\title{
Long-Term Changes in Physical Activity Following a One-Year Home-Based Physical Activity Counseling Program in Older Adults with Multiple Morbidities
}

\author{
Katherine S. Hall, ${ }^{1}$ Richard Sloane, ${ }^{2,3}$ Carl F. Pieper, ${ }^{2,3,4}$ Matthew J. Peterson, ${ }^{1,2,3}$ \\ Gail M. Crowley, ${ }^{5}$ Patricia A. Cowper, ${ }^{6}$ Eleanor S. McConnell, ${ }^{1,2,7}$ Hayden B. Bosworth, $, 8,9$ \\ Carola C. Ekelund, ${ }^{1}$ and Miriam C. Morey ${ }^{1,2,3,9}$
}

${ }^{1}$ Geriatric Research, Education, and Clinical Center, Durham Veterans Affairs Medical Center, 508 Fulton Street, Durham, NC 27705, USA

${ }^{2}$ Center for the Study of Aging and Human Development, Duke University, Durham, NC 27710, USA

${ }^{3}$ Claude D. Pepper Older Americans Independence Center, Duke University, Durham, NC 27710, USA

${ }^{4}$ Department of Biostatistics and Bioinformatics, Duke University, Durham, NC 27710, USA

${ }^{5}$ Neurodiagnostic Center, Durham Veterans Affairs Medical Center, 508 Fulton Street, Durham, NC 27705, USA

${ }^{6}$ Duke Clinical Research Institute, Duke University Medical Center, Durham, NC 27705, USA

${ }^{7}$ School of Nursing, Duke University, Durham, NC 27710, USA

${ }^{8}$ Health Services Research and Development Service, Durham Veterans Affairs Medical Center, 508 Fulton Street, Durham, NC 27705, USA

${ }^{9}$ Department of Medicine, Duke University, Durham, NC 27710, USA

Correspondence should be addressed to Katherine S. Hall, katherine.hall3@va.gov

Received 25 August 2010; Revised 16 November 2010; Accepted 6 December 2010

Academic Editor: Iris Reuter

Copyright (C) 2011 Katherine S. Hall et al. This is an open access article distributed under the Creative Commons Attribution License, which permits unrestricted use, distribution, and reproduction in any medium, provided the original work is properly cited.

This study assessed the sustained effect of a physical activity (PA) counseling intervention on PA one year after intervention, predictors of sustained PA participation, and three classes of post-intervention PA trajectories (improvers, maintainers, and decliners) in 238 older Veterans. Declines in minutes of PA from 12 to 24 months were observed for both the treatment and control arms of the study. PA at 12 months was the strongest predictor of post-intervention changes in PA. To our surprise, those who took up the intervention and increased PA levels the most, had significant declines in post-intervention PA. Analysis of the three post-intervention PA trajectories demonstrated that the maintenance group actually reflected a group of nonresponders to the intervention who had more comorbidities, lower self-efficacy, and worse physical function than the improvers or decliners. Results suggest that behavioral counseling/support must be ongoing to promote maintenance. Strategies to promote PA appropriately to subgroups of individuals are needed.

\section{Introduction}

The number of studies targeting physical activity (PA) behavior in older adults has increased markedly over the past decade. Such studies have largely been effective at increasing PA among older adult participants, and have demonstrated comparable results across home-based and center-based formats $[1,2]$. Relatively little is known about the extent to which intervention effects are maintained over the long term, although results from previous research suggest that recidivism upon cessation of intervention is a reality across a variety of populations and behaviors $[1,3-8]$. While studies examining adherence at shorter time points (e.g., 6 months) are more common, long-term followups in older adults remain sparse [9]. As a result, strategies to foster PA adherence over the long term in an aging population remain to be identified. To date, much of the research surrounding PA has focused on identifying strategies to increase adoption. 
While the evidence supporting the beneficial effects of a physically active lifestyle on chronic disease management is vast [10], similar to pharmacologic therapies, the benefits of PA are directly proportional to adherence rates. Thus, behavioral researchers and public health officials must now also consider the challenge of developing effective strategies to facilitate PA maintenance.

In addition to post-intervention trends, studies on the individual characteristics that determine long-term patterns of adherence are needed. Previous research suggests that psychosocial factors and previous PA behavior are significantly associated with higher PA levels in older adults $[6,7,11,12]$ However, the role of factors such as comorbidity, physical function, and physical performance in determining longterm PA maintenance or decline in older adults remains unclear. Thus, it is unclear whether there may be subgroups of individuals who respond differentially to the cessation of a behavioral intervention. Examining how PA levels change following an intervention and identifying the characteristics of those who benefit the most and least is a necessary and important precursor to developing targeted evidence-based programs that promote long-term activity adherence in older adults.

The Veterans LIFE study $[13,14]$ is a 12 -month randomized controlled trial of PA telephone counseling (PAC) to increase PA in older veterans. One year after finishing the study, attempts were made to contact all participants to participate in followup data collection. The first aim of the study was to examine PA levels during a 12-month nonintervention period and determine the effect of participation in the intervention on post-intervention changes. The second aim was to examine behavioral and psychosocial predictors of PA during the post-intervention period. The third aim was to identify three classes of post-intervention PA trajectories: maintenance, gains, and losses and explore the behavioral, functional and psychosocial characteristics of these three groups.

\section{Methods}

A complete description of the Veterans LIFE study has been reported elsewhere [13]. In brief, this study was a randomized controlled trial comparing a 1-year multicomponent physical activity counseling (PAC) program with usual care (UC). The Durham Veterans Affairs Institutional Review Board reviewed and approved the research protocol, and written consent was obtained from all participants.

Participants in this study were older male patients followed at the Durham Veterans Affairs Medical Center (VAMC) primary care clinics. To participate, patients had to be 70 years of age or greater, able to walk a short distance without human assistance, not regularly participate in PA, not suffer from dementia or severe hearing/vision loss, and be free of serious or terminal medical conditions that would preclude safe engagement in PA.

398 patients were recruited to participate in a 12-month randomized, controlled PAC intervention. Participants were randomized to one of two groups at baseline: PAC $(n=199)$ or UC $(n=199)$. The PA objectives for the PAC group were to walk or perform lower extremity physical activity for 30 minutes or more on 5 or more days of the week and to perform 15 minutes of lower extremity strength training on 3 days each week. UC consisted of usual care received within the context of visits to primary care providers within the same time frame.

Guided by social cognitive theory [15] and the transtheoretical model of behavior change [16], the PAC consisted of baseline physical activity counseling, telephone counseling, endorsement of the study by the patient's primary care provider, automated telephone messaging from the primary care provider, and individualized progress reports. The PAC intervention components and CONSORT diagram illustrating participant flow across the 1-year life of the study have been discussed in detail elsewhere [13]. This study resulted in significant improvements in functional performance and PA in the PAC group but not the UC group [13].

To determine whether participation in the Veterans LIFE study resulted in sustained behavior change 12 months after intervention and identify factors that predict behavior change, attempts were made to recontact all participants one year after finishing the program to ask them to complete measures on-site at the Durham VAMC. Of the 199 men randomized to PAC, 177 completed the study and 123 gave consent for the followup assessment; resulting in a $70 \%$ followup response rate. Of the 199 men randomized to UC, 176 completed the study and 115 gave consent for the followup assessment; resulting in a $65 \%$ followup response rate. 116 individuals did not return for the 24month followup data collection.

\section{Measures}

3.1. Physical Activity. We measured minutes of moderateintensity endurance PA and minutes of moderate-intensity strength PA using the Community Healthy Activities Model Program for Seniors (CHAMPS) [17, 18]. The CHAMPS questionnaire assesses the duration of a range of physical activities from which moderate activities can be separated. Minutes of endurance PA were calculated as the sum of brisk walking, running/jogging, cycling/stationary cycle, and aerobic machine items from the CHAMPS. Minutes of strength PA were calculated as the sum of moderate/heavy weight lifting, light strength training, and general conditioning items from the CHAMPS.

3.2. Physical Performance. Rapid gait speed (meters/second) was assessed over two trials of an 8 -foot walk test.

3.3. Self-Efficacy. Two items were used to assess self-efficacy separately for walking/endurance activities and strength training activities; the content of these items was created to be consistent with the Veterans LIFE study counseling. The first question asked participants, "How sure are you that you could walk or do another type of endurance exercise for 30 minutes or more on five or more days of the week? The 30 minutes do not have to be all at the same time." The second item asked, "How sure are you that you could do exercises for 15 minutes, three days a week, to make your 
legs stronger?" Responses for these two items ranged from 1 (not at all confident) to 5 (extremely confident). A scale score was created by taking the average of the responses on the two items.

3.4. Comorbidities. Number of chronic conditions was assessed using the Older Americans Resources and Services survey (OARS) [19], which surveys 35 medical conditions.

3.5. Physical Function. Self-rated physical function was assessed using the physical function subscale of the Medical Outcomes Study 36-item Short-Form Health Survey (SF-36) [20]. Scores range from $0-100$, with higher scores reflecting better physical function.

\section{Statistical Analysis}

4.1. Aim 1: Intervention Effects on Post-Intervention Changes in PA. To measure the effect of the PAC intervention on activity levels one year after completion of the study, we compared the activity levels of those formerly in the PAC and UC groups. Differences in minutes of moderateintensity endurance PA and minutes of moderate-intensity strength PA between the two groups were tested using ordinary least squares, adjusting for 0-12-month change, 12month PA status, and age, race, education, and number of comorbidities all measured at baseline.

4.2. Aim 2: Behavioral and Psychosocial Predictors of PostIntervention $P A$. The association of behavioral and psychosocial factors with post-intervention change in PA was assessed using multiple regression analysis. We identified behavioral and psychosocial factors that in the literature are associated with physical activity behavior change in older adults: PA (endurance and strength PA at Month 12), exercise self-efficacy (Month 12), self-reported physical function (Month 12), and rapid gait speed (Month 12). Two a priori regression models containing all five predictor factors were run; one with 12-24 month change in endurance PA as the outcome variable, and one with 12-24 month change in strength PA as the outcome variable. Each model controlled for age, education level, and number of comorbidities. Diagnostics of model fit were run on each model to assess collinearity.

4.3. Aim 3: Evaluating Three Classes of PA Trajectories in the PAC Group. We classified individuals from the PAC group as maintainers, improvers, or decliners based upon their changes in PA levels from 12 to 24 months. In creating these categories we were cognizant of two issues: (1) we wanted the "Improve" and "Decline" categories to capture changes in PA behavior that were sizeable and reflected a purposeful increase or decrease in effort, and (2) we wanted the "Maintenance" category to have a similar buffer to minimize the risk of categorizing individuals as improvers or maintainers who had not substantively changed their PA behavior after intervention. Thus, group membership was based on the change in moderate-intensity endurance and strength PA from Month 12 to Month 24 using the 12-month median value for each variable as the criterion.

The 12-month median value for endurance PA was 45 minutes. Thus, improvement in minutes of moderateintensity endurance PA was defined as an increase greater than 45 minutes/week from 12 to 24 months, while decline was defined as any negative change greater than -45 minutes/week. Individuals were classified as maintainers when the change in endurance PA minutes from 12 to 24 months was less than or equal to \pm 45 minutes/week.

The 12-month median value for strength PA was 75 minutes. Thus, improvement in minutes of moderateintensity strength PA was defined as an increase greater than 75 minutes/week from 12 to 24 months, while decline was defined as any negative change greater than -75 minutes/week. Individuals were classified as maintainers when the change in strength PA minutes from 12 to 24 months was less than or equal to \pm 75 minutes/week.

We also examined whether those who maintained, improved, or declined PA from 12 to 24 months differed significantly on the same behavioral and psychosocial factors identified in Section 4.2. We used a series of pairwise comparisons to determine whether meaningful differences in these characteristics existed between the three classes. As is commonly the case with exploratory analyses, caution is warranted when interpreting the pairwise comparisons as they do not correct for Type I error. We conducted all analyses using SAS Version 9.1 (SAS Inc., Cary, NC).

\section{Results}

Demographic characteristics have been reported previously [13]. To summarize, study participants were older men ( $M$ age $=77$ years, Range $=70-92$ years) of mixed educational backgrounds, with $26 \%$ receiving a college degree and $45 \%$ reporting a high school graduate equivalency or less. Study participants reported approximately five chronic conditions $(M \pm$ SD; $5.15 \pm 2.44)$.

As mentioned previously, $30 \%$ of the PAC group and $35 \%$ of the UC group did not complete the followup data collection. Thus, to determine whether these response trends introduced any bias into our results, we first compared those who completed 24-month followup $(n=238)$ and those who did not $(n=115)$ on treatment arm, demographic characteristics (i.e., age, race, education level), number of comorbidities, minutes of endurance PA, minutes of strength PA, rapid gait speed, and physical function. The only significant differences $(P<.05)$ between those lost to followup and those retained were on race and rapid gait speed. Those who completed data collection at Month 24 were more likely to be white and have a faster rapid gait speed than those who did not complete data collection at 24 months.

5.1. Intervention Effects on Post-Intervention Changes in PA. Among those study participants who completed assessments at 24 months, participants in the PAC group reported more minutes of moderate-intensity endurance PA and moderateintensity strength PA per week at 12 months compared to 
TABLE 1: Minutes of strength and endurance PA at 12 and 24 months by intervention group in participants who completed the 24-month followup.

\begin{tabular}{lcccccc}
\hline & $\begin{array}{c}\text { Minutes of } \\
\text { Endurance PA } \\
12 \text { months }\end{array}$ & $\begin{array}{c}\text { Minutes of } \\
\text { Endurance PA } \\
24 \text { months }\end{array}$ & $\begin{array}{c}\text { Minutes of } \\
\text { Strength PA } \\
12 \text { months }\end{array}$ & $\begin{array}{c}\text { Minutes of } \\
\text { Strength PA } \\
\text { 24 months }\end{array}$ & $\begin{array}{c}\text { Adjusted Mean-Level } \\
\text { Change of Endurance } \\
\text { PA 12 to 24 Months }\end{array}$ & $\begin{array}{c}\text { Adjusted Mean-Level } \\
\text { Change of Strength } \\
\text { PA 12 to 24 Months }\end{array}$ \\
\hline $\begin{array}{l}\text { PA Counseling } \\
(n=123)\end{array}$ & $74.6(10.3)$ & $52.4(9.2)$ & $55.8(5.5)$ & $37.3(6.1)$ & -10.4 & -12.0 \\
$\begin{array}{l}\text { Usual Care } \\
(n=114)\end{array}$ & $44.7(10.7)$ & $43.2(9.6)$ & $29.5(5.7)$ & $33.8(6.3)$ & -14.3 & -4.5 \\
\hline
\end{tabular}

Values represent Means and Standard Errors.

the UC group (Table 1). As expected, after finishing the intervention, minutes of moderate-intensity endurance PA and minutes of moderate-intensity strength PA declined for both groups. Although the declines in endurance PA were greater among the PAC group, they did not differ significantly from those observed in the UC group $(\beta=21.5$, $P=.22)$; the decline in minutes of strength $P A$, however, was significantly greater in the PAC group $(\beta=23.1, P=.01)$. Despite these declines over the last 12 months, minutes of moderate-intensity endurance PA and minutes of moderateintensity strength PA remained higher among those in the PAC group compared to the UC group.

\subsection{Behavioral and Psychosocial Predictors of Post-Interven-} tion PA. Results of the multiple regression analysis indicated that the predictor variables accounted for $51.6 \%$ of the variance in post-intervention change in endurance PA. Minutes of endurance PA at Month $12(\beta=-0.87)$, minutes of strength PA at Month $12(\beta=0.26)$, self-efficacy $(\beta=20.62)$, physical function $(\beta=0.96)$, and rapid gait speed $(\beta=$ -47.82) were all significant predictors of post-intervention change in endurance PA. Collinearity diagnostics indicated high levels of collinearity between 12-month endurance $\mathrm{PA}$ and 12-24-month endurance PA change. A subsequent regression model in which 12-month endurance PA was excluded as a predictor variable resulted in a much better fitting model. However, this model indicated no significant effects for any of the other factors and accounted for only $3.0 \%$ of the variance in post-intervention change in endurance PA; demonstrating that previous endurance PA is the most important determinant of post-intervention changes in endurance PA.

Relative to post-intervention change in strength PA, results of the multiple regression analysis indicated that the predictor variables accounted for $33.2 \%$ of the variation. Minutes of strength PA at Month $12(\beta=-0.59)$, minutes of endurance PA at Month $12(\beta=0.14)$, and self-efficacy $(\beta=$ 11.81) were all significant predictors of post-intervention change in endurance PA. Collinearity diagnostics indicated low levels of collinearity between 12-month strength PA and 12-24-month strength PA change. Although collinearity among model variables was low, we determined a priori to run parallel models for endurance and strength; testing a second regression model in which 12-month strength PA was excluded as a predictor variable. Although this model provided a much better fit, no significant effects for any of the other factors were observed and this model accounted for only $7.6 \%$ of the variance in post-intervention change in strength PA. These results demonstrate that previous strength PA is the most important determinant of postintervention changes in strength PA.

\subsection{Group Characteristics of Long-Term Maintenance, Im-} provement or Decline. Figure 1(a) shows the three classes for post-intervention changes in minutes of moderate-intensity endurance PA for the PAC group. Individuals who improved their minutes of endurance PA from 12 to 24 months $(n=$ 22) had moderate rates of endurance PA at Month $12(M=$ $47.3, \mathrm{SD}=62.8$ minutes $/$ week). Individuals who declined in minutes of endurance PA from 12 to 24 months $(n=39)$ had higher rates of endurance PA at Month $12(M=178.4$, $\mathrm{SD}=155.0$ minutes/week). Individuals who maintained their minutes of endurance PA from 12 to 24 months $(n=62)$ had the lowest rates of endurance PA at Month 12 compared to the other categories ( $M=22.8, \mathrm{SD}=67.9$ minutes/week).

Figure 1(b) shows the three PA trajectories for postintervention changes in minutes of moderate-intensity strength PA for the PAC group. Individuals who improved their minutes of strength PA from 12 to 24 months $(n=16)$ had moderate rates of strength PA at Month $12(M=54.7$, $\mathrm{SD}=59.4$ ). Individuals who declined in minutes of strength PA from 12 to 24 months $(n=38)$ had higher rates of strength PA at Month $12(M=101.8, \mathrm{SD}=59.1)$. Individuals who maintained their minutes of strength PA from 12 to 24 months $(n=69)$ had the lowest rates of strength PA at Month 12 compared to the other categories $(M=30.3$, $\mathrm{SD}=33.9)$.

Characteristics of long-term maintenance, improvement or decline of minutes of moderate-intensity endurance PA are shown in Table 2. As expected, minutes of endurance PA at Month 12 was the most consistent discriminant of post-intervention changes in endurance PA. Specifically, the pairwise comparison analyses demonstrated that individuals who declined from 12 to 24 months had significantly $(P<$ .05) greater levels of endurance PA to start with (at Month 12) compared to those in the improve and maintenance groups. In addition to 12-month endurance PA, minutes of strength PA, number of comorbidities, self-efficacy, and rapid gait speed at 12 months were also significantly different across the three groups $(P s<.05)$. However, these variables only significantly discriminated those in the maintenance group from the other two groups. Specifically, individuals 


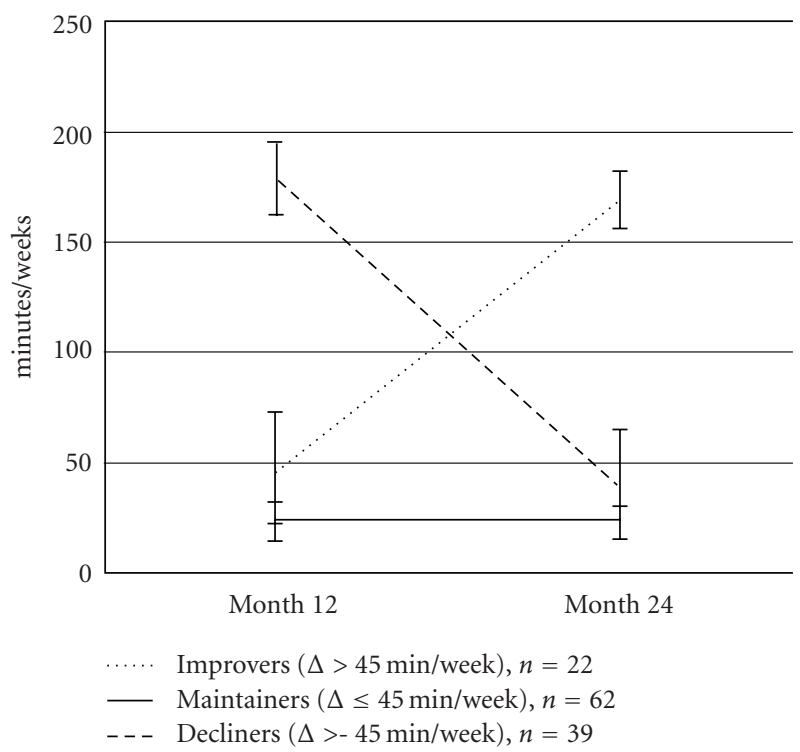

(a)

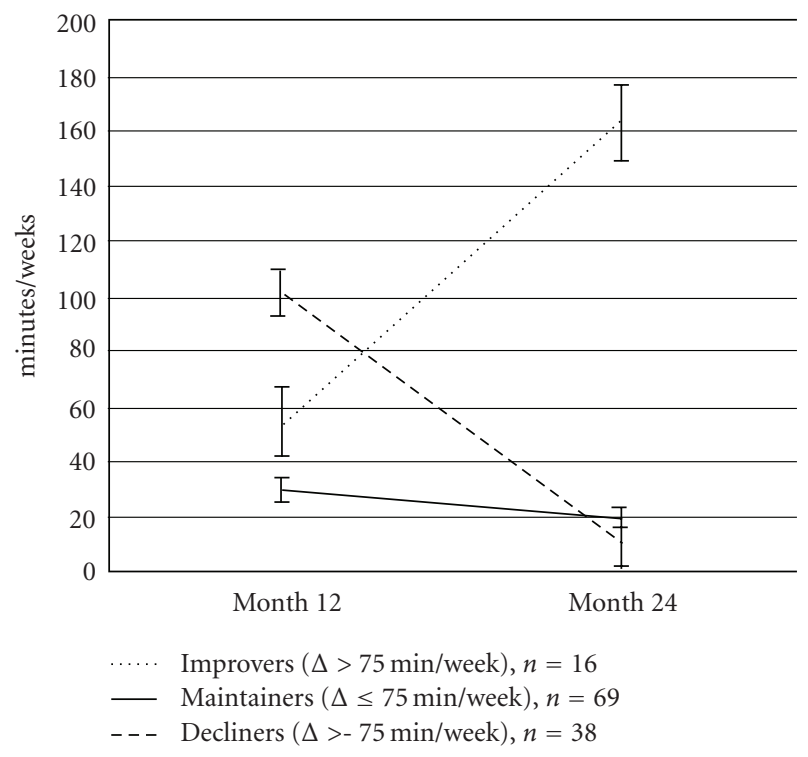

(b)

Figure 1: (a) Trajectories of post-intervention changes in minutes of moderate-intensity endurance PA. (b) Trajectories of postintervention Changes in Minutes of Moderate-Intensity Strength PA (Note that values represent means and standard errors).

who maintained their minutes of endurance PA over the 12-month post-intervention period had the lowest rate of activity at 12 months, had more comorbidities, were less efficacious for physical activity, reported worse physical function, and had significantly slower gait speed compared to those in the improve or decline groups $(P<.05)$.

Characteristics of long-term maintenance, improvement or decline of minutes of strength PA are shown in Table 3. As expected, minutes of strength PA at Month 12 was the most consistent discriminant of post-intervention changes in strength PA. Specifically, the pairwise comparison analyses demonstrate that individuals who declined from 12 to 24 months had significantly $(P<.05)$ greater levels of strength PA to start with (at Month 12) compared to those in the improve or maintenance groups. In addition to 12-month strength PA, only self-efficacy at 12 months significantly differed across the three groups; with individuals who maintained their minutes of strength PA over the 12-month after intervention period being significantly less efficacious for physical activity compared to those in the improve or decline groups $(P<.05)$.

\section{Discussion}

The literature is replete with research describing interventions aimed at improving PA; with many of these interventions using behavioral theory-based approaches to modify PA [21-25]. Although declines in PA can be expected following cessation of intervention, there is hope that some behavioral benefit from PA interventions can be sustained beyond the intervention period. In this study we sought to determine if there was any maintenance of PA following a year of no contact with former study participants. As expected, given the cessation of all study-related contact and resources, minutes of moderate-intensity endurance and strength PA among PAC participants decreased following the cessation of the home-based PA counseling program. However, despite these post-intervention declines in the PAC group, minutes of endurance PA and minutes of strength PA at Month 24 remained higher than baseline PA levels (data not shown). Moreover, PA rates in the PAC group remained higher than those in the UC group at 24 months, suggesting some long-term benefit of PA counseling on PA behavior compared to usual care.

Consistent with our expectations and previous reports in the literature, PA at 12 months was the single most important predictor of post-intervention change in activity levels. However, higher levels of PA at Month 12 were significantly associated with decreases in PA over the postintervention period. These results suggest that altering behavior in the short term is not sufficient, in and of itself, to promote behavior maintenance. Indeed, upon cessation of the intervention and the resources and support associated with it, older adults who were successful in changing their behavior during the intervention were the most vulnerable to post-intervention declines. These results underscore the importance of on-going support following a behavioral intervention to improve maintenance and reduce the likelihood of regressing back to a sedentary lifestyle. Strategies to promote maintenance should be a systematic component of any behavioral intervention and warrant future study.

Self-efficacy, physical function, and gait speed also demonstrated significant effects on post-intervention changes in PA. However, PA at Month 12 accounted for a major portion of the variance in post-intervention change in PA, such that in the absence of the 12-month measure of PA, no significant effects were observed for any of these other candidate predictors. These results, coupled with the nonsignificant bivariate associations (data not shown) observed between post-intervention change in PA and 
TABLE 2: Characteristics of maintenance, improvement, and decline from 12 to 24 months: minutes of moderate-intensity endurance PA.

\begin{tabular}{|c|c|c|c|c|c|c|}
\hline Variable & $\begin{array}{l}\text { Maintainers } \\
\qquad(n=62) \\
M(\mathrm{SD})\end{array}$ & $\begin{array}{c}\text { Improvers } \\
(n=22) \\
M(\mathrm{SD})\end{array}$ & $\begin{array}{c}\text { Decliners } \\
(n=39) \\
M(\mathrm{SD})\end{array}$ & $\begin{array}{c}\text { Improvers } \\
\text { versus } \\
\text { Maintainers } \\
P \text {-value }\end{array}$ & $\begin{array}{l}\text { Maintainers } \\
\text { versus } \\
\text { Decliners } \\
P \text {-value }\end{array}$ & $\begin{array}{c}\text { Improvers } \\
\text { versus } \\
\text { Decliners } \\
P \text {-value }\end{array}$ \\
\hline $\begin{array}{l}\text { Weekly minutes of } \\
\text { endurance PA }\end{array}$ & $23.5(63.8)$ & $47.3(62.8)$ & $178.4(155)$ & .35 & $<.0001^{*}$ & $<.0001^{*}$ \\
\hline $\begin{array}{l}\text { Weekly minutes of } \\
\text { strength PA }\end{array}$ & $41.9(59.6)$ & $79.3(47.4)$ & $63.9(50)$ & $.01^{*}$ & $.05^{*}$ & .29 \\
\hline Age & $80.0(4.7)$ & $78.6(5.1)$ & $78.3(4.4)$ & .26 & .09 & .79 \\
\hline \multicolumn{7}{|l|}{ Race } \\
\hline White & $45(73 \%)$ & $17(77 \%)$ & $23(59 \%)$ & \multirow{2}{*}{.32} & \multirow{2}{*}{.09} & \multirow{2}{*}{.15} \\
\hline Others & $17(27 \%)$ & $5(23 \%)$ & $16(41 \%)$ & & & \\
\hline \multicolumn{7}{|l|}{ Education } \\
\hline$\leq$ H.S. grad & $26(42 \%)$ & $11(50 \%)$ & $16(41 \%)$ & \multirow{3}{*}{.82} & \multirow{3}{*}{.84} & \multirow{3}{*}{.97} \\
\hline Some college & $17(27 \%)$ & $4(18 \%)$ & $15(39 \%)$ & & & \\
\hline$\geq$ College & $19(31 \%)$ & $7(32 \%)$ & $8(21 \%)$ & & & \\
\hline Comorbidity & $5.4(2.5)$ & $4.5(2.2)$ & $4.4(2.4)$ & .14 & .054 & .92 \\
\hline Self-Efficacy & $6.7(2.2)$ & $8.0(1.4)$ & $8.0(1.6)$ & $.01 *$ & $.0009^{*}$ & .93 \\
\hline $\begin{array}{l}\text { Physical function } \\
\text { (SF-36) }\end{array}$ & $62.3(21.9)$ & $75.7(20.9)$ & $80.0(16.9)$ & $.01^{*}$ & $<.0001^{*}$ & .45 \\
\hline $\begin{array}{l}\text { Gait velocity rapid } \\
\mathrm{m} / \mathrm{s}\end{array}$ & $1.61(0.47)$ & $1.89(0.35)$ & $1.89(0.41)$ & $.01^{*}$ & $<.01^{*}$ & .96 \\
\hline
\end{tabular}

All variables assessed at Month $12 .{ }^{*}$ Significant at $P<.05$

any of the predictor variables (with the exception of PA at 12 months), suggest that these associations are not independent of PA at 12 months. That these pathways may be indirect is consistent with a social cognitive perspective in which behavior influences, and is influenced by, individual cognitions and abilities [15] and has been demonstrated in previous research with older adults $[6,7]$.

In an effort to better understand the individual variability following post-intervention behavior and develop tailored clinical interventions to promote physical activity, we conducted extensive exploratory analyses. Based upon their post-intervention changes in PA, individuals were categorized according to three trajectory classes: improvers, maintainers, or decliners. We chose these three groups a priori, knowing that recipients of the intervention increased PA during the intervention period [13] and expecting that recipients would fall into three categories during the nonintervention period: improvers, maintainers, or decliners. However, upon examination of the changes in PA during the intervention across the three groups (data not shown) we discovered that these group descriptors did not accurately reflect the trends in our data. Those who increased their minutes of PA postintervention demonstrated small improvements during the intervention. In contrast, those who substantially decreased their minutes of PA post-intervention were those who had made the most gains during the intervention. To our surprise, those who maintained their minutes of PA post-intervention appear to not have taken to the intervention at all. Thus, it appears that in this study the individuals most likely to maintain their activity levels post-intervention were those who abstained from making any changes to their behavior at all over the course of an intervention and would be more accurately described as nonresponders. Despite this group comprising nearly $50 \%$ of the PAC arm, previous analyses report significant improvements in PA during the intervention [13]; underscoring the need for individual-level analyses to detect and characterize these underlying patterns of behavior.

In addition to characterizing the post-intervention change patterns in PA, we had hoped to identify characteristics that would differentiate those who improve, nonrespond/maintain, or decline in post-intervention PA; selecting factors that could be used in clinical care to identify individuals who may need additional support or booster shots (e.g., self-efficacy, physical function, and physical performance). In this sample of older adults, the nonresponders/maintainers had more comorbidities, lower selfefficacy to continue exercising, lower physical function, and slower gait speed than either the improvers or decliners. This pattern of results, together with the significantly lower level of PA at 12 months seen in the nonresponders/maintainers, suggests that the nonresponders/maintainers have physical, functional, and psychosocial disadvantages that make them even less likely to respond to an intervention that is largely home based and unsupervised.

In a previous PA clinical trial of individuals undergoing three months of supervised exercise followed by six months of home-based exercise, we observed significant improvements in fitness and physical function; largely driven by changes occurring during the supervised period. These 
TABle 3: Characteristics of maintenance, improvement, and decline from 12 to 24 months: minutes of moderate-intensity strength PA.

\begin{tabular}{|c|c|c|c|c|c|c|}
\hline Variable & $\begin{array}{c}\text { Maintainers } \\
\quad(n=69) \\
M(\mathrm{SD})\end{array}$ & $\begin{array}{c}\text { Improvers } \\
(n=16) \\
M(\mathrm{SD})\end{array}$ & $\begin{array}{c}\text { Decliners } \\
(n=38) \\
M(\mathrm{SD})\end{array}$ & $\begin{array}{l}\text { Improvers } \\
\text { versus } \\
\text { Maintainers } \\
P \text {-value }\end{array}$ & $\begin{array}{c}\text { Maintainers } \\
\text { versus } \\
\text { Decliners } \\
P \text {-value }\end{array}$ & $\begin{array}{c}\text { Improvers } \\
\text { versus } \\
\text { Decliners } \\
P \text {-value }\end{array}$ \\
\hline $\begin{array}{l}\text { Weekly minutes of } \\
\text { endurance PA }\end{array}$ & $65.5(105.1)$ & $113.4(208.9)$ & $82.2(104.5)$ & .14 & $.05^{*}$ & .92 \\
\hline $\begin{array}{l}\text { Weekly minutes of } \\
\text { strength PA }\end{array}$ & $30.3(33.9)$ & $54.8(59.4)$ & $101.8(59.1)$ & .06 & $<.0001^{*}$ & $<.01^{*}$ \\
\hline Age & $79.5(4.8)$ & $79.0(4.8)$ & $78.7(4.5)$ & .68 & .36 & .81 \\
\hline \multicolumn{7}{|l|}{ Race } \\
\hline White & $47(68 \%)$ & $11(69 \%)$ & $27(71 \%)$ & \multirow{2}{*}{.95} & \multirow{2}{*}{.79} & \multirow{2}{*}{.87} \\
\hline Others & $22(32 \%)$ & $5(31 \%)$ & $11(29 \%)$ & & & \\
\hline \multicolumn{7}{|l|}{ Education } \\
\hline$\leq$ H.S. grad & $28(41 \%)$ & $6(38 \%)$ & $19(50 \%)$ & \multirow{3}{*}{.36} & \multirow{3}{*}{.15} & \multirow{3}{*}{.21} \\
\hline Some college & $20(29 \%)$ & $4(25 \%)$ & $12(32 \%)$ & & & \\
\hline$\geq$ College & $21(30 \%)$ & $6(38 \%)$ & $7(18 \%)$ & & & \\
\hline Comorbidity & $5.0(2.4)$ & $4.6(2.3)$ & $5.0(2.7)$ & .54 & .98 & .55 \\
\hline Self-Efficacy & $6.9(2.1)$ & $8.4(1.9)$ & $7.7(1.6)$ & $<.01^{*}$ & $.04^{*}$ & .20 \\
\hline $\begin{array}{l}\text { Physical function } \\
\text { (SF-36) }\end{array}$ & $67.5(23.3)$ & $77.2(17.6)$ & $72.5(19.8)$ & .11 & .25 & .47 \\
\hline $\begin{array}{l}\text { Gait velocity rapid } \\
\mathrm{m} / \mathrm{s}\end{array}$ & $1.71(0.50)$ & $1.82(0.25)$ & $1.78(0.43)$ & .42 & .50 & .77 \\
\hline
\end{tabular}

All variables assessed at Month $12 .{ }^{*}$ Significant at $P<.05$

improvements regressed upon transition to home-based exercise [26]. Subsequent analyses indicated that nonadherence to home-based PA could be traced back to low levels of PA during the supervised portion of the trial. Physical function, physical performance, comorbidity, and symptoms were also related to nonadherence in the home setting [27]. These studies, along with our results here, suggest that we can identify characteristics of individuals who may be poor candidates for home-based interventions and who require on-going support once an intervention has ended.

We expected that factors such as self-efficacy, physical function, and physical performance might distinguish between the improvers and the decliners. However, none of the variables examined proved fruitful. Instead, the differences between these two trajectories rest solely on initial (Month 12) PA levels. This may be due, in part, to the relatively small sample size in each group and concomitant lack of statistical power. These results have implications for future PA interventions, however, for they suggest that consistent with health promotion guidelines $[3,28]$, individuals who are active at high levels at the end of an intervention are in need of extended resources and support if these changes are to be sustained.

Our decision to classify participants as maintainers, decliners, or improvers in the manner that we did may be unique. Unlike other studies that have assessed success or failure to long-term PA adherence relative to meeting activity guidelines $[3,11,29,30]$, here, success was defined relative to the individual; thus taking into account individual limitations and barriers. Although our PA counseling was directed towards meeting national goals of 150 minutes per week or more of PA, we recognized that this goal was not achievable for many of our participants and endorse the call for older adults with comorbidities to be as physically active as they are able.

Our study has some limitations that must be considered when interpreting these results. First, although every attempt was made to collect 24-month followup data from all of the study participants, and thus minimize drop-out bias, we were unable to do so. However, we did attempt to address this limitation by examining the differences between those who completed measures at 24 months and those who declined participation at 24 months. These analyses suggest that our sample may be biased toward the more physically active and better functioning. However, no significant betweengroup differences were reported by treatment arm or on other dimensions of health status or self-reported physical function. Second, we recognize that one possible explanation for the pattern of PA change observed here may be attributed to regression to the mean. However, we believe that the significant effects of the intervention demonstrated previously in the PAC group [13] indicate recidivism following the end of an intervention, likely due to the removal of intervention supports and expectations, and not due to some random effect as implied by regression to the mean. Third, although we included variables that demonstrated significant associations with PA in previous studies $[3,7,29,31]$, we acknowledge that our models did not include other intrapersonal, interpersonal, or environmental factors which may influence behavior change.

Despite calls for more studies of long-term PA participation and maintenance $[9,29,32]$, we have much to learn, 
underscoring the difficulty in understanding (and altering) PA, a complex behavior. However, in this study we did identify a number of important factors which are related to changes in PA behavior over the long term. One of these factors, self-efficacy, is a modifiable construct and as such, is worthy of more investigation. Future studies of long-term maintenance that consider other variables such as environmental supports and barriers are needed. Importantly, our results also suggest that home-based PA may be of limited benefit to certain groups of lower functioning and highly sedentary older adults. Thus, similar to strategies to increase adoption and maintenance of PA, tailored approaches that target at-risk populations and accommodate individual, functional, and behavioral barriers may also be needed for sustained behavior change.

\section{Acknowledgments}

This study was supported by Grants from the Veterans Affairs Rehabilitation Research and Development (no. E3386R), Morey PI, and the National Institutes of Health (AG028716), Morey Co-PI. H. Bosworth is supported by a Department of Veterans Affairs Health Services Research and Development Research Career Scientist Award (RCS 08-027). The authors wish to also acknowledge the dedication of our research staff members: Megan Pearson, Jennifer Chapman, and Teresa Howard and to thank the participants of the Veterans LIFE Study. The views expressed by the authors do not necessarily reflect the views of the Department of Veterans Affairs.

\section{References}

[1] C. M. Castro and A. C. King, "Telephone-assisted counseling for physical activity," Exercise and Sport Sciences Reviews, vol. 30, no. 2, pp. 64-68, 2002.

[2] N. L. Ashworth, K. E. Chad, E. L. Harrison, B. A. Reeder, and S. C. Marshall, "Home versus center based physical activity programs in older adults," Cochrane Database of Systematic Reviews, vol. 1, 2005.

[3] E. M. Hertogh, Y. Vergouwe, A. J. Schuit, P. H. M. Peeters, and E. M. Monninkhof, "Behavioral changes after a 1-yr exercise program and predictors of maintenance," Medicine and Science in Sports and Exercise, vol. 42, no. 5, pp. 886-892, 2010.

[4] J. K. Vallance, K. S. Courneya, R. C. Plotnikoff, I. Dinu, and J. R. MacKey, "Maintenance of physical activity in breast cancer survivors after a randomized trial," Medicine and Science in Sports and Exercise, vol. 40, no. 1, pp. 173-180, 2008.

[5] M. L. Greaney, D. Riebe, C. E. Garber et al., "Long-term effects of a stage-based intervention for changing exercise intentions and behavior in older adults," Gerontologist, vol. 48, no. 3, pp. 358-367, 2008.

[6] E. McAuley, G. J. Jerome, S. Elavsky, D. X. Marquez, and S. N. Ramsey, "Predicting long-term maintenance of physical activity in older adults," Preventive Medicine, vol. 37, no. 2, pp. 110-118, 2003.

[7] E. McAuley, K. S. Morris, R. W. Motl, L. Hu, J. F. Konopack, and S. Elavsky, "Long-term follow-up of physical activity behavior in older adults," Health Psychology, vol. 26, no. 3, pp. 375-380, 2007.

[8] H. E. Whitson, M. T. Heflin, and B. M. Burchett, "Patterns and predictors of smoking cessation in an elderly cohort," Journal of the American Geriatrics Society, vol. 54, no. 3, pp. 466-471, 2006.

[9] L. R. Brawley, W. J. Rejeski, and A. C. King, "Promoting physical activity for older adults: the challenges for changing behavior," American Journal of Preventive Medicine, vol. 25, no. 3, supplement 2, pp. 172-183, 2003.

[10] Physical Activity Guidelines Advisory Committee, "Physical Activity Guidelines Advisory Committee Report," Washington, DC, USA: U.S. Department of Health and Human Services, 2008.

[11] A. C. King, M. Kiernan, R. F. Oman, H. C. Kraemer, M. Hull, and D. Ahn, "Can we identify who will adhere to long-term physical activity? Signal detection methodology as a potential aid to clinical decision making," Health Psychology, vol. 16, no. 4, pp. 380-389, 1997.

[12] R. F. Oman and A. C. King, "Predicting the adoption and maintenance of exercise participation using self-efficacy and previous exercise participation rates," American Journal of Health Promotion, vol. 12, no. 3, pp. 154-161, 1998.

[13] M. C. Morey, M. J. Peterson, C. F. Pieper et al., "The veterans learning to improve fitness and function in elders study: a randomized trial of primary care-based physical activity counseling for older men: clinical investigations," Journal of the American Geriatrics Society, vol. 57, no. 7, pp. 1166-1174, 2009.

[14] M. C. Morey, M. J. Peterson, C. F. Pieper et al., "Project LIFE-learning to improve fitness and function in elders: methods, design, and baseline characteristics of randomized trial," Journal of Rehabilitation Research and Development, vol. 45, no. 1, pp. 31-42, 2008.

[15] A. Bandura, "Self-efficacy: toward a unifying theory of behavioral change," Psychological Review, vol. 84, no. 2, pp. 191-215, 1977.

[16] J. O. Prochaska and W. F. Velicer, "The transtheoretical model of health behavior change," American Journal of Health Promotion, vol. 12, no. 1, pp. 38-48, 1997.

[17] A. L. Stewart, K. M. Mills, P. G. Sepsis et al., "Evaluation of champs, a physical activity promotion program for older adults," Annals of Behavioral Medicine, vol. 19, no. 4, pp. 353361, 1997.

[18] A. L. Stewart, K. M. Mills, A. C. King, W. L. Haskell, D. Gillis, and P. L. Ritter, "CHAMPS physical activity questionnaire for older adults: outcomes for interventions," Medicine and Science in Sports and Exercise, vol. 33, no. 7, pp. 1126-1141, 2001.

[19] G. G. Fillenbaum, Multidimensional Functional Assessment of Older Adults, Lawrence Erlbaum Associates, Hillsdale, NJ, USA, 1988.

[20] J. E. Ware and C. D. Sherbourne, "The MOS 36-item shortform health survey (SF-36). I. Conceptual framework and item selection," Medical Care, vol. 30, no. 6, pp. 473-483, 1992.

[21] S. Wilcox, M. Dowda, L. C. Leviton et al., "Active for Life. Final Results from the Translation of Two Physical Activity Programs," American Journal of Preventive Medicine, vol. 35, no. 4, pp. 340-351, 2008.

[22] B. Reger, L. Cooper, S. Booth-Butterfield et al., "Wheeling Walks: a community campaign using paid media to encourage walking among sedentary older adults," Preventive Medicine, vol. 35, no. 3, pp. 285-292, 2002.

[23] M. A. Napolitano, J. A. Whiteley, G. Papandonatos et al., "Outcomes from the women's wellness project: a communityfocused physical activity trial for women," Preventive Medicine, vol. 43, no. 6, pp. 447-453, 2006. 
[24] M. D. Litt, A. Kleppinger, and J. O. Judge, "Initiation and maintenance of exercise behavior in older women: predictors from the social learning model," Journal of Behavioral Medicine, vol. 25, no. 1, pp. 83-97, 2002.

[25] R. A. Fielding, J. Katula, M. E. Miller et al., "Activity adherence and physical function in older adults with functional limitations," Medicine and Science in Sports and Exercise, vol. 39, no. 11, pp. 1997-2004, 2007.

[26] M. C. Morey, M. Schenkman, S. A. Studenski et al., "Spinalflexibility-plus-aerobic versus aerobic-only training: effects of a randomized clinical trial on function in at-risk older adults," Journals of Gerontology: Series A Biological Sciences and Medical Sciences, vol. 54, no. 7, pp. M335-M342, 1999.

[27] M. C. Morey, P. M. Dubbert, M. E. Doyle et al., "From supervised to unsupervised exercise: factors associated with exercise adherence," Journal of Aging and Physical Activity, vol. 11, no. 3, pp. 351-368, 2003.

[28] D. M. Jacobson, L. Strohecker, M. T. Compton, D. L. Katz, and M. Barry, "Physical activity counseling in the adult primary care setting: position statement of the American College of Preventive Medicine," American Journal of Preventive Medicine, vol. 29, no. 2, pp. 158-162, 2005.

[29] A. C. King, B. Marcus, D. Ahn et al., "Identifying subgroups that succeed or fail with three levels of physical activity intervention: the activity counseling trial," Health Psychology, vol. 25, no. 3, pp. 336-347, 2006.

[30] M. Mänty, A. Heinonen, R. Leinonen et al., "Long-term effect of physical activity counseling on mobility limitation among older people: a randomized controlled study," Journals of Gerontology - Series A Biological Sciences and Medical Sciences, vol. 64, no. 1, pp. 83-89, 2009.

[31] D. Riebe, B. J. Blissmer, M. L. Greaney, C. Ewing Garber, F. D. Lees, and P. G. Clark, "The relationship between obesity, physical activity, and physical function in older adults," Journal of Aging and Health, vol. 21, no. 8, pp. 1159-1178, 2009.

[32] B. H. Marcus, L. H. Forsyth, E. J. Stone et al., "Physical activity behavior change: issues in adoption and maintenance," Health Psychology, vol. 19, supplement 1, pp. 32-41, 2000. 


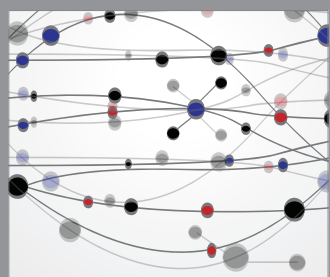

The Scientific World Journal
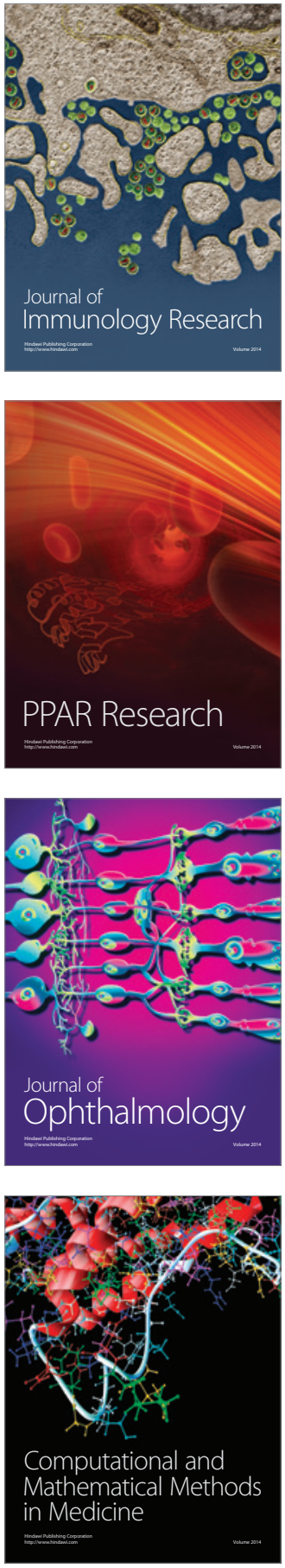

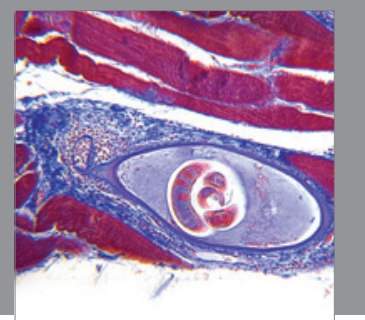

Gastroenterology

Research and Practice
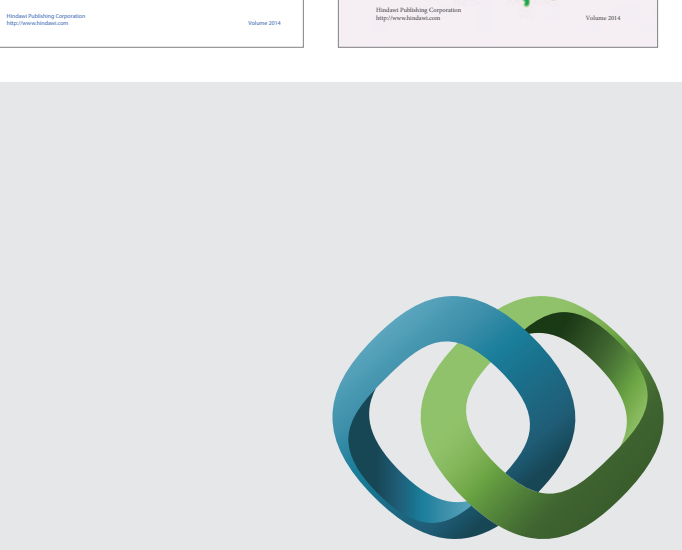

\section{Hindawi}

Submit your manuscripts at

http://www.hindawi.com
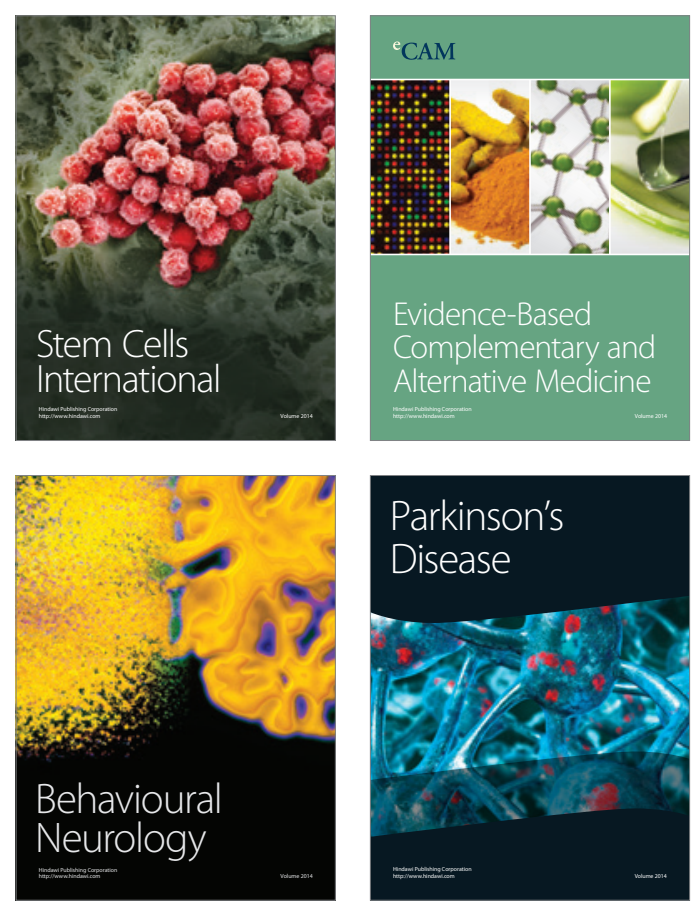

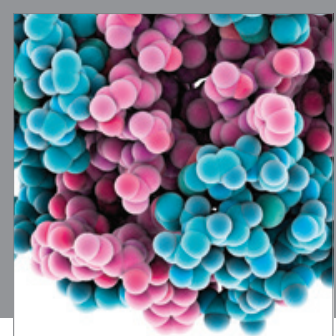

Journal of
Diabetes Research

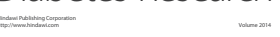

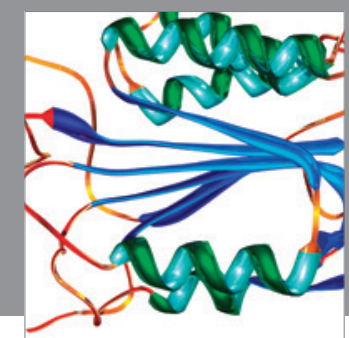

Disease Markers
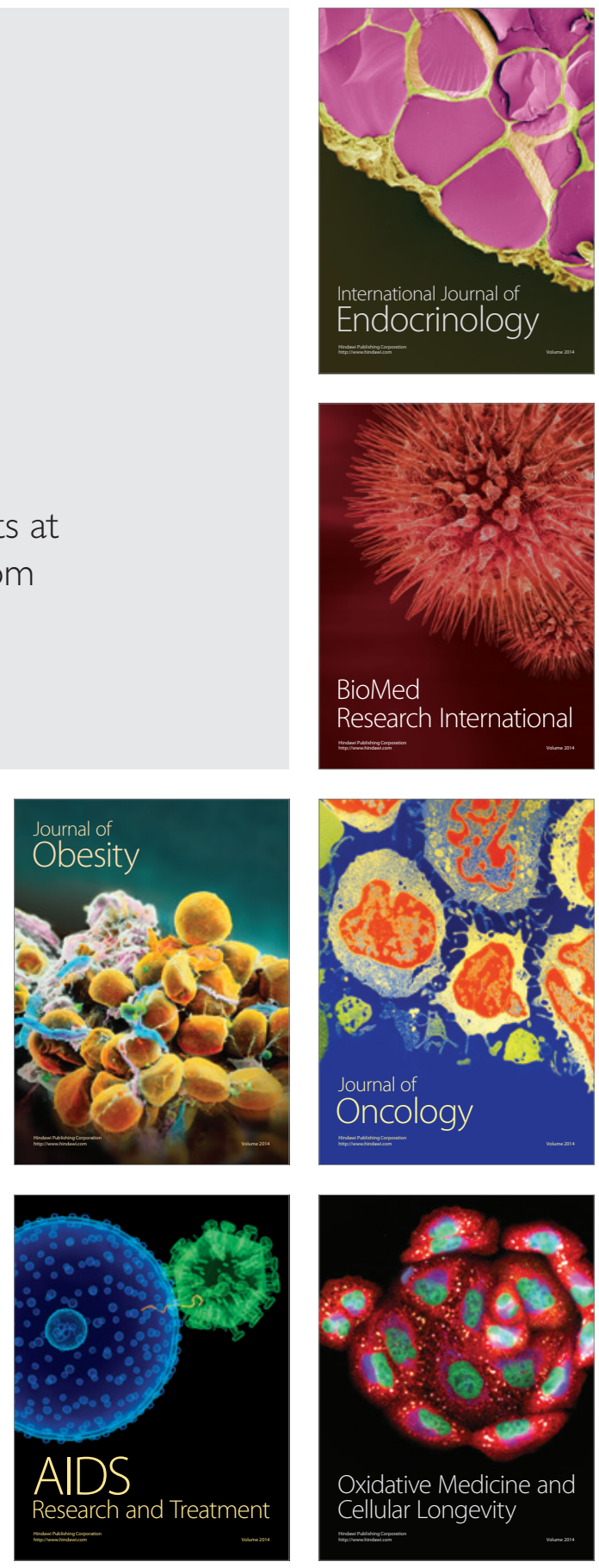